\title{
Diffusion Tensor Imaging MRI for Chronic Pain Management
}

\author{
Tae Shin Kim, Jung Yul Park ${ }^{凶}$ \\ Department of Neurosurgery, Korea University Medical Center Anam Hospital, Seoul, Korea
}

\begin{abstract}
Chronic pain syndromes are associated with neural reorganization in specific regions associated with perception, processing, and integration of pain. This is especially true in neuropathic pain. Neuroimaging methods have identified various structures in the brain involved in the processing of pain and its control. Diffusion tensor imaging (DTI), a magnetic resonance imaging-based method, allows in vivo mapping of the anatomical connections in the human brain. Although this modality is relatively new in pain research filed authors believe that it will provide useful information regarding the connectivity between various pain centers in brain, brainstem and spinal cord with pain processing pathways that may correlate the types, location and severity of pain experienced by patients. Hence, authors tried to search and review the basics of DTI, with its advancement, and clinically applied studies to determine its usefulness in chronic pain management.
\end{abstract}

Key Words: Chronic pain; Diffusion tensor imaging; Magnetic resonance image.

$\triangle$ Corresponding author: Jung Yul Park, Department of Neurosurgery, College of Medicine, Korea University Anam Hospital, 73 Inchon-ro, Seongbuk-gu, Seoul 02841, Korea. Tel: 82-2-920-5729, Fax: 82-2-929-0629, E-mail: jypark98@korea.ac.kr

\section{INTRODUCTION}

In 1990's, since Michael Moseley reported that water diffusion of white matter was anisotropic, many different attempts to visualize direction of white matter fibers were followed and nowadays these tries came up to visualize each white matter fiber tract by connecting white matter fiber directions ${ }^{16)}$. Diffusion tensor imaging (DTI) MRI is a technique that can show white matter fiber directions. Many efforts to apply this technique to various treatment options are being made. Motor function related fiber tracking and language related fiber tracking techniques have been and still are used frequently for brain tumor surgeries but applying this technique for pain management is still in very early stage.

Chronic pain occurs due to damage of diverse pain related fiber tract or interruption of physiologic pathway to central nervous system. Pain related fiber tracts are well demonstrated anatomically in traditional experimental method, but tracing it in living human body has not been clearly demonstrated. With current technology and advancement of scientific knowledge, it would be theoretically possible to trace these pain related fiber tracts using DTI MRI and visualize them to 3D images called tractography. Thus, with this technique, it would be possible to construct images that connect pain related pathway from brain cortex to spinal nerve1 and find location of damaged site and of nucleus that controls or conveys pain. In this review, authors tried to review basics and experiences, both experimental and clinical, which applied DTI images for pain management, how it can be applied and how it can be used to figure out the damaged site. Further, discussion on possible future role of DTI in the management of chronic pain is described.

\section{MRI DTI}

DTI is a MRI technique that can deduct microstructural information by measuring directionality and magnitude of water diffusion. DTI comprises a group of calculated eigenvalues $(\lambda 1$, $\lambda 2, \lambda 3)$ and eigenvectors $(\varepsilon 1, \varepsilon 2, \varepsilon 3)$ of diffusion properties of a tissue. The sum of the eigenvalues $(\lambda 1+\lambda 2+\lambda 3)$ is called the trace, while their average (=trace/ 3 ) is called the mean diffusivity (MD) or apparent diffusion coefficient (ADC) $)^{2,8)}$.

Fractional anisotropy (FA) is an index for the amount of diffusion asymmetry. The formula of FA is as listed below.

$$
\mathrm{FA}=\sqrt{\frac{\left(\lambda_{1}-\lambda_{2}\right)^{2}+\left(\lambda_{2}-\lambda_{3}\right)^{2}+\left(\lambda_{1}-\lambda_{3}\right)^{2}}{2\left(\lambda_{1}{ }^{2}+\lambda_{2}{ }^{2}+\lambda_{3}{ }^{2}\right)}}
$$


The value of $F A$ varies between 0 and 1 . If the diffusion ellipsoid is a sphere, $\lambda 1=\lambda 2=\lambda 3$ and FA $=0$. With progressive diffusion anisotropy, the eigenvalues become more unequal, the ellipsoid becomes more elongated, and the FA $\rightarrow 1^{2}$.

These two measures MD, FA are commonly used to evaluate microstructure variation of tissue (Fig. 1$)^{1)}$.

$\mathrm{MD}$ or ADC (Apparent diffusion coefficient) expresses net degree of displacement of water molecules. If we express MD on anatomical images, high MD shows white appearance and low MD shows dark appearance ${ }^{8}$. Increasing structures in cell impedes water diffusion, so in structure-increasing state, displacement of water molecules decreases and MD decreases also. Therefore, in state of white matter maturation, high degree myelination, cell proliferation in neoplasia, MD decreases. In situation of demyelination, axon degeneration, edema, $\mathrm{MD}$ increases ${ }^{2}$.

FA expresses anisotrophy of water molecular diffusion. Higher the FA, higher the integrity of water molecules is and it means that water molecules are densely packed. Therefore, in state of white matter maturation, or of high degree myelination, FA increases. In state of demyelination or axon degeneration, FA decreases. In hundreds of studies, FA decreased in disease situation $^{2}$. If FA is visualized on anatomical image, high FA shows white appearance and low FA shows dark appearance.

Tractography is a technique that makes this anisotropy to a 3D model. By connecting the orientation of the predominant direction of diffusion, reconstructing the major white matter tract can be possible ${ }^{1)}$.

There have been several attempts to trace the alteration of FA, $\mathrm{MD}$ in chronic pain patients, and trials for detecting nucleus involved in pain tract, and stimulating nucleus to management pain are on process. With more studies and experiences in future it would be possible to have clear $3 \mathrm{D}$ reconstructed DTI images in patients with various chronic pain states thus will be helpful and useful in determining the locations, degrees, and severities of injury that correlates with the symptoms and signs from each patient. In this regard, it will not only aid in chronic pain management but also will provide the information on natural course of each painful condition and on changes following treatments. Moreover, as previous studies have shown that DTI in motor pathway showed usefulness on prediction of recovery following injuries, it can provide to physician and to patient very important aspect of chronic pain management, the prognosis and future progression of pain states that are currently not possible to determine.

\section{TRACING PAIN TRACT CONNECTION WITH MRI DTI}

Previous articles have shown several nerve fiber pathways related with pain. The regions thought to be involved in the chronic pain pathways include pre-frontal cortex, anterior cingulate cortex (ACC), medial dorsal nucleus (MDN) of the thalamus, ventral posterior nucleus (VPN) of the thalamus, insula, primary sensory cortex, and globus pallidus ${ }^{6}$. Also., several other regions are reported to be involved using with DTI techniques.

Previous works expected putamen may be activated during pain, but it has been thought that this nucleus responds to pain due to motor processing to make person avoid or reduce pain. DTI tractography showed structural connection between pain activated putaminal regions and several other pain related pathway that are mentioned above ${ }^{15}$.

The reason why this is important is that if more tracts that are related with pain are found, safer target that can be controlled by stimulation can be found. Stimulating ventral caudal thalamus and periaqueductal gray has been a method for controlling chronic pain nowadays, but the putamen is an easier target

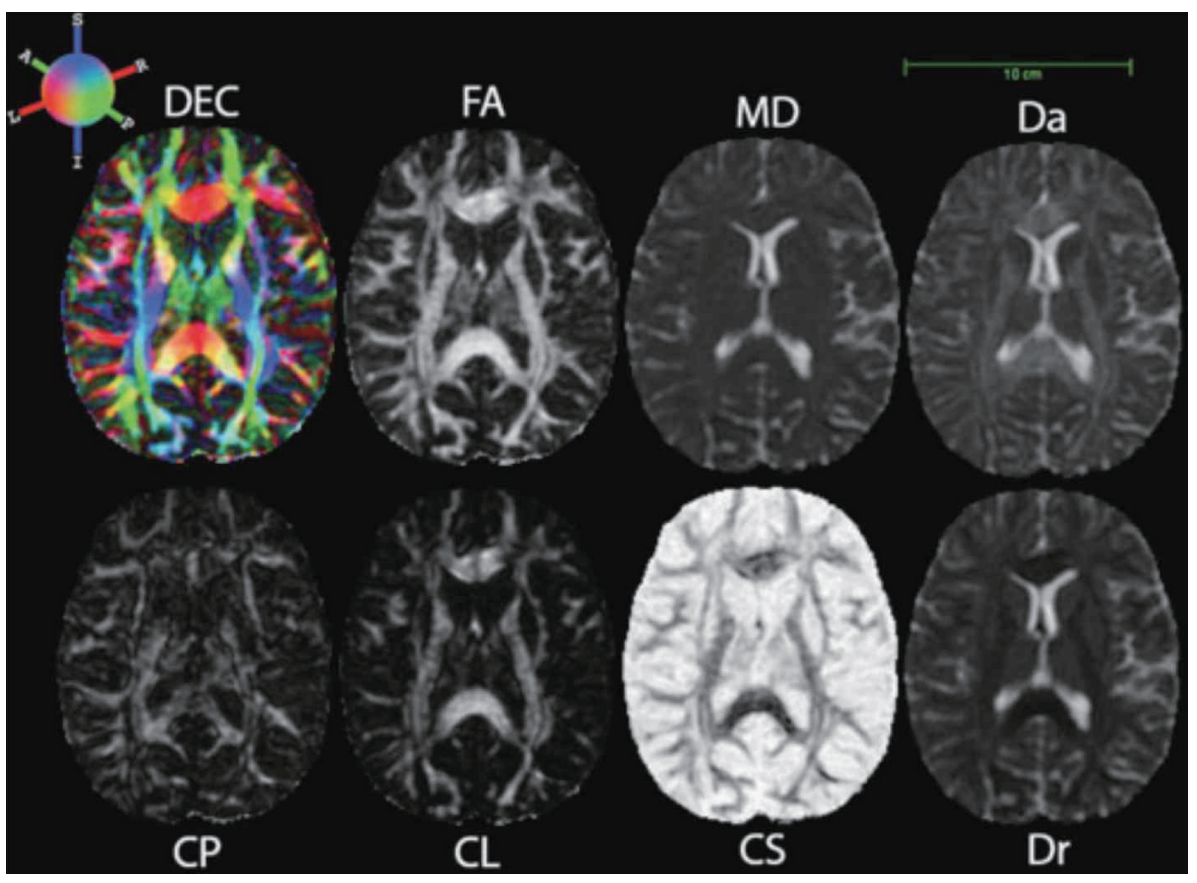

Fig. 1. Diffusion tensor imaging (DTI) stain maps from a single DTI data set (Adopted from Alexander AL, et al. ${ }^{1)}$ with permission). 
to reach since it is located more lateral than other brain targets. So using DTI to find out the connection between nucleus and tracts can help understanding the mechanism of pain.

\section{APPLICATIONS OF DTI TO MANAGEMENT OF CHRONIC PAIN SYNDROMES}

Many therapeutic approaches including chronic motor cortex stimulation, and deep brain stimulation (DBS) to the periventricular/periaqueductal gray (PAG/PVG) and sensory thalamus are processed but failed to sustain relief of pain with high visual analogue scale (VAS) score rating pain patients).

Recently, DBS implantation after planning with DTI tractography has been described ${ }^{9}$. The spinothalamocortical (STC) tract is regarded as a tract responsible for transmission of thalamic pain. Therefore, the thalamus or the posterior limb of the internal capsule has been thought to be ideal targets for DBS in patient with chronic pain. But conventional MRI has limitations for showing STC tract. In this regard, DTI tractography can show STC tract directly and provide guidance for planning target area for stimulation. DBS implantation guided by DTI images is proved to have advantage for precise targeting and have better outcome than done only by conventional MRI.

Other studies have shown that DBS in the PAG/PVG area has produced more controlling effect than DBS in the thalamic ventroposterolateral (VPL) nucleus in patients who suffer neuropathic pain ${ }^{3,12)}$. The PAG/PVG is known to be associated with spinomesencephalic tract which is fibers that synapse with laminae I, IV, V and VI, and terminate in PAG area. Owen at al. ${ }^{13)}$ reported a case of patient who underwent left lower leg amputation and suffered from phantom limb pain and relieved from pain after DBS in the PAG/PVG region. After the surgery, the author used tractography to observe fiber pathways of region that each contact points of electrodes are placed. By visualizing tracts, contact points which are located in the region where tract that connects affective dimension of pain matrix had most treatment effect. So planning the insertion site of DBS electrode can be possible with DTI images.

Conventional MRI has a limitation to identify thalamic nuclei in detail but MRI DTI can show the expected location of nuclei by combining several related fiber tracts. Kovanlikaya et al. ${ }^{10)}$ performed DTI tractography for patients who were waiting for DBS, targeting the thalamic VPL nucleus. Thalamic-somatosensory, thalamic-somatomotor and dentato-rubro-thalamic connecting fibers were visulalized in fiber tractography images, and they were chosen for guidance to estimate the location of the VPL nuclei of the thalamus (Fig. 2) ${ }^{10)}$. Preoperative DTI provided more chance to identify patient-specific targets and to enhance the safety and efficacy of DBS surgery.

\section{APPLICATIONS OF DTI TO SPINAL CORD TRACT}

Conventional MRI has played an important role in diagnosing spinal cord lesion. However, several spinal cord injury patients have discrepancy between conventional MRI findings and clinical presentation. Many trials have been made to compensate these differences, however MRI DTI can be one useful choice because it can express microstructure changes (Fig. 3, 4) ${ }^{7,14}$.

Recently, there have been several trials to compare ADC and FA values of each cord level and ,of these, Song et al. ${ }^{14)}$ checked $\mathrm{ADC}$ and FA values of each cervical cord levels of patients who have cervical compressive myelopathy. As a result, FA value of compressed cord has decreased and ADC value has increased compared to non-compressed cord. Budzik et al. ${ }^{4)}$ performed DTI on patients who have cervical spondylotic myelopathy and compared the conformity degree of clinical assessment with DTI, and with T2 MRI. The study showed there is a positive correlation between decrease in FA and functional impairment of the upper and lower limbs.

Mansour et al. ${ }^{11)}$ followed patients over a one year who had a single episode of subacute back pain. Authors divided those patients into two groups, one group who had recovered, and the other group who had persisted pain into chronicity. MRI DTI was implemented and authors compared FA difference and checked where the abnormal region on FA image is linked on tractography. As a result, there were multiple regional FA decreases in the patients who had persisted pain. It seems to be more sensitive than T2 imaging and have more correlation with clinical symptoms.

Fig. 2. Fiber tractography of patient : Thalamocortical sensory fiber tracts (Yellow), Dentato-ruboro-thalamic-cortical fiber tracts (Red; red arrow), and corticothalamic motor fiber tracts (Blue) are shown. These fiber tracts are chosen for estimating the location of the VPL and the ventral intermedius nuclei of the thalamus. The distal tip of the DBS electrode (Purple, purple arrow) located in the ventrodorsolateral nucleus (Adopted from Kovanlikaya I, et al. ${ }^{10)}$ with permission).
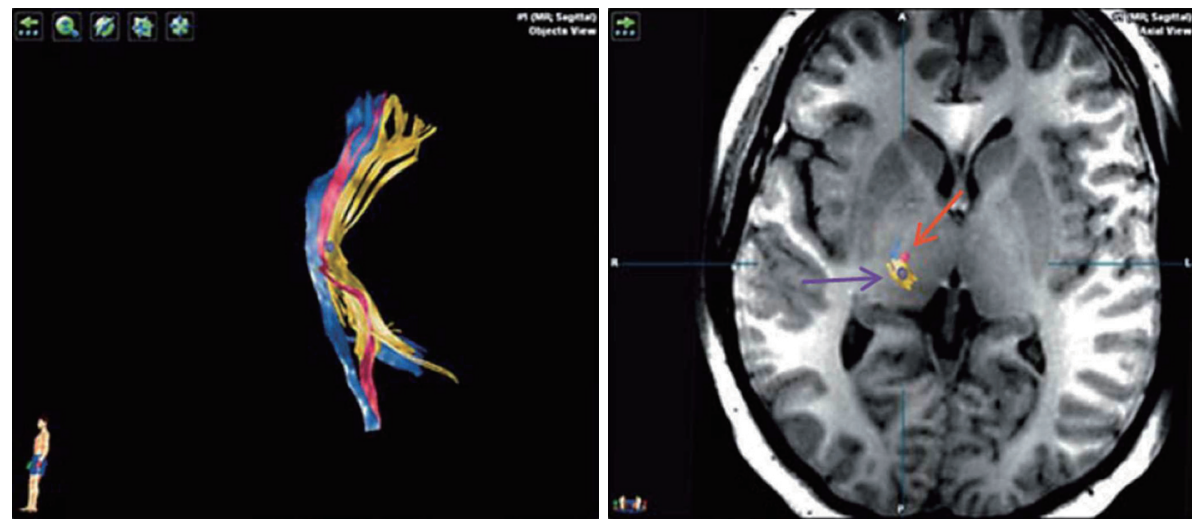

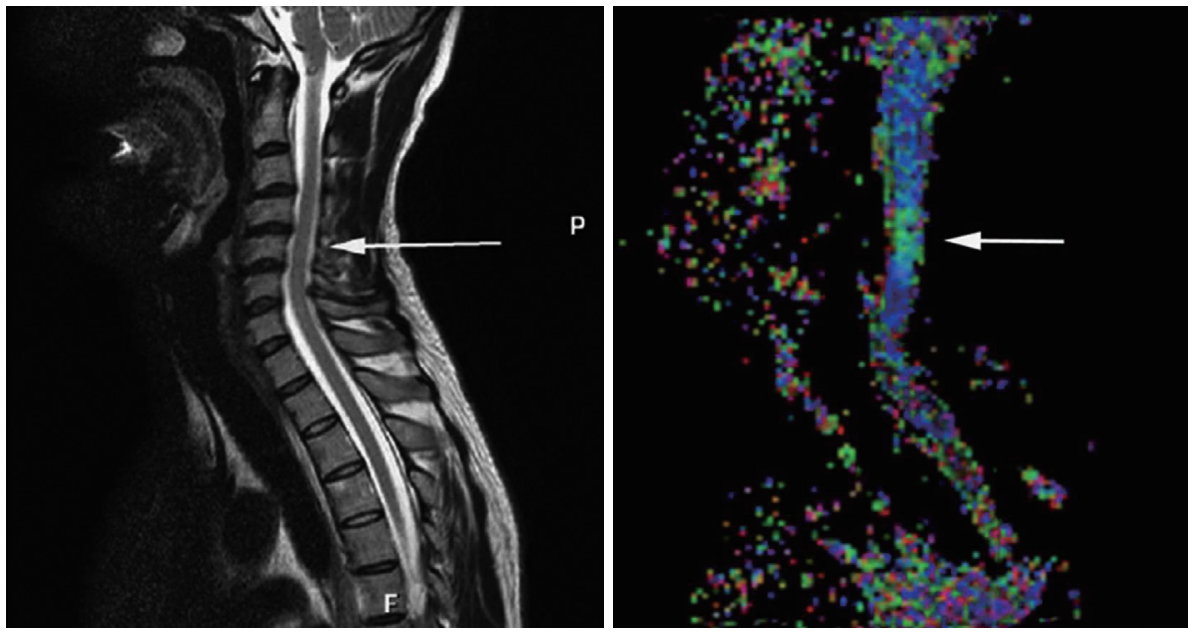

Fig. 3. Comparison of Conventional MRI T2WI image and DTI map of the same patient showing no abnormal signal intensity on MRI T2WI image but showing abnormal green signal intensity on DTI map (Adopted from Song $\mathrm{T}$, et al. ${ }^{14)}$ with permission).

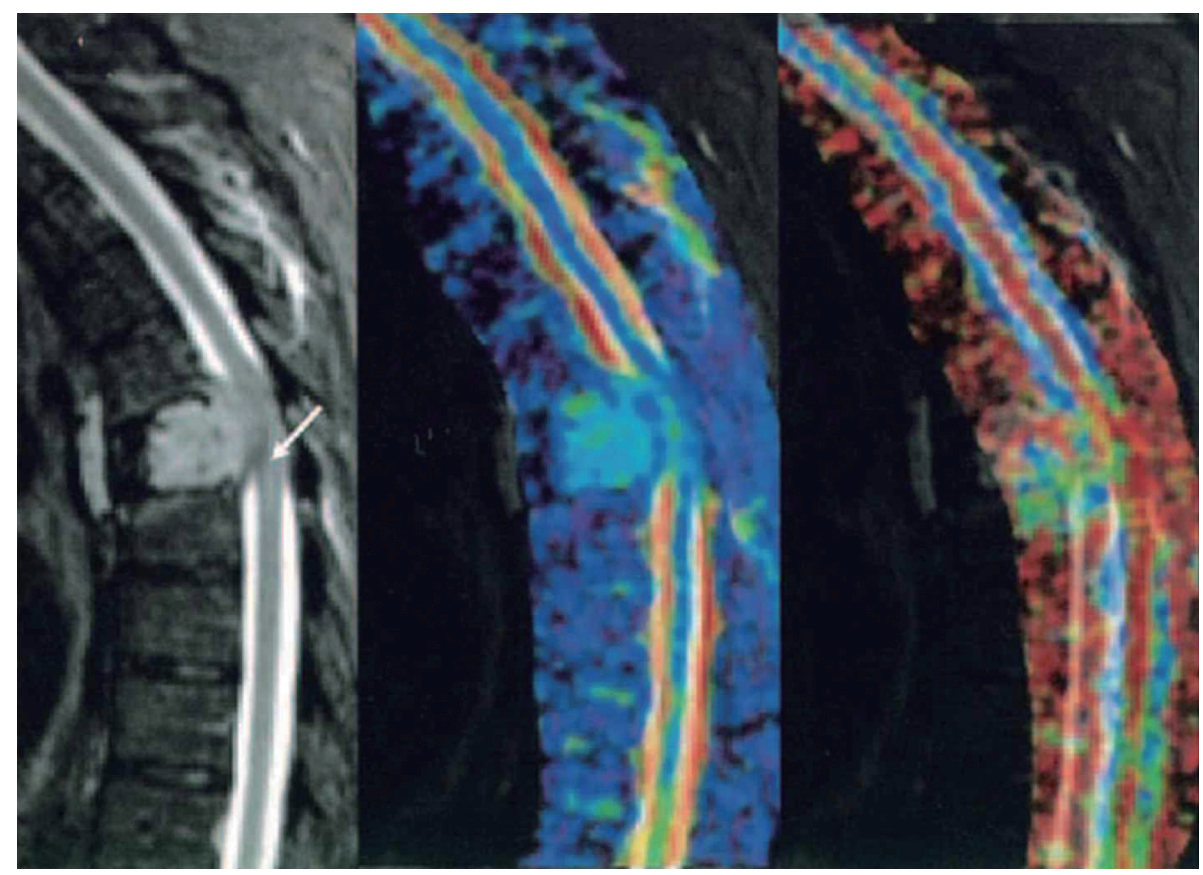

Fig. 4. MR imaging of a spinal cord compression due to a breast tumor metastasis. Images are from C7-T10. Colorcoded scale related to parameters values. Higher values are coded in red, medium values in green, and lower values in blue. T2 weighted imaging (first image) shows a high signal intensity (white arrow), ADC (second image) is slightly increased (green area) on same area, and FA (third image) is decreased (green area) (Adopted from Falcon $D$, et al. ${ }^{7)}$ with permission).

\section{CONCLUSION}

Recent studies, although few in number, have shown that current technique of MRI DTI can provide more understanding about connection of pain fibers. Studies focusing on abnormal regions in pain suffering patients have shown to be complex and still on progress. Standard and conventional imaging studies have been greatly improved and play a pivotal role in diagnosis of various chronic pain syndromes. However, because of its inherent limitations, DTI can be a useful tool to provide additional information about pain pathway connection status for more accurate diagnosis and prediction of prognosis. With more basic and clinical studies in future, DTI will provide a new horizon in the field of chronic pain management.

\section{REFERENCES}

1. Alexander AL, Hurley SA, Samsonov AA, Adluru N, Hosseinbor AP, Mossahebi $\mathrm{P}$, et al. : Characterization of cerebral white matter properties using quantitative magnetic resonance imaging stains. Brain Connect 1 : 423-446, 2011

2. Alexander AL, Lee JE, Lazar M, Field AS : Diffusion tensor imaging of the brain. Neurotherapeutics $4: 316-329,2007$

3. Bittar RG, Burn SC, Bain PG, Owen SL, Joint C, Shlugman D, et al. : Deep brain stimulation for movement disorders and pain. J Clin Neurosci 12 : 457-463, 2005

4. Budzik JF, Balbi V, Le Thuc V, Duhamel A, Assaker R, Cotten A : Diffusion tensor imaging and fibre tracking in cervical spondylotic myelopathy. Eur Radiol 21 : 426-433, 2011

5. Coenen VA, Kieselbach K, Mader I, Reinacher PC : Diffusion tensor magnetic resonance imaging (DTI) tractography-guided deep brain stimulation in neuropathic pain. Acta Neurochir (Wien) 157 : 739-741, 2015

6. Ellingson BM, Mayer E, Harris RJ, Ashe-McNally C, Naliboff BD, Labus JS, et al. : Diffusion tensor imaging detects microstructural reorganization in the brain associated with chronic irritable bowel syndrome. Pain 154 : 1528-1541, 2013

7. Facon D, Ozanne A, Fillard P, Lepeintre JF, Tournoux-Facon C, Ducreux $\mathrm{D}: \mathrm{MR}$ diffusion tensor imaging and fiber tracking in spinal cord compression. AJNR Am J Neuroradiol 26 : 1587-1594, 2005

8. Feldman HM, Yeatman JD, Lee ES, Barde LH, Gaman-Bean S : Diffusion tensor imaging : a review for pediatric researchers and clinicians. J Dev 
Behav Pediatr 31 : 346-356, 2010

9. Hunsche S, Sauner D, Runge MJ, Lenartz D, El Majdoub F, Treuer H, et al. : Tractography-guided stimulation of somatosensory fibers for thalamic pain relief. Stereotact Funct Neurosurg 91 : 328-334, 2013

10. Kovanlikaya I, Heier L, Kaplitt M : Treatment of chronic pain : diffusion tensor imaging identification of the ventroposterolateral nucleus confirmed with successful deep brain stimulation. Stereotact Funct Neurosurg 92 : 365-371, 2014

11. Mansour AR, Baliki MN, Huang L, Torbey S, Herrmann KM, Schnitzer TJ, et al. : Brain white matter structural properties predict transition to chronic pain. Pain 154 : 2160-2168, 2013

12. Owen SL, Green AL, Stein JF, Aziz TZ: Deep brain stimulation for the alle- viation of post-stroke neuropathic pain. Pain $120: 202-206,2006$

13. Owen SL, Heath J, Kringelbach ML, Stein JF, Aziz TZ : Preoperative DTI and probabilistic tractography in an amputee with deep brain stimulation for lower limb stump pain. Br J Neurosurg 21 : 485-490, 2007

14. Song T, Chen WJ, Yang B, Zhao HP, Huang JW, Cai MJ, et al. : Diffusion tensor imaging in the cervical spinal cord. Eur Spine J 20 : 422-428, 2011 Tomycz ND, Friedlander RM : The experience of pain and the putamen : a new link found with functional MRI and diffusion tensor imaging. Neurosurgery 69 : N12-N13, 2011

15. Turner R, Le Bihan D, Maier J, Vavrek R, Hedges LK, Pekar J : Echo-planar imaging of intravoxel incoherent motion. Radiology 177 : 407-414, 1990 